\title{
Eligibility for the shorter regimen for multidrug-resistant tuberculosis in Mexico
}

To the Editor:

We read with interest the articles by Sotgiu et al. [1] and Dalcolmo et al. [2], which initiated a debate on the suitability of the shorter regimen for multidrug-resistant (MDR) tuberculosis (TB) cases in different settings [2-6]. The shorter World Health Organization (WHO) regimen [7, 8] is composed of an initial phase of 4-6 months of kanamycin, moxifloxacin, prothionamide, clofazimine, pyrazinamide, high-dose isoniazid and ethambutol, followed by 5 months of moxifloxacin, clofazimine, pyrazinamide and ethambutol.

In summary, $\mathrm{TB}$ cases not previously treated with second-line anti-TB drugs are eligible for the shorter regimen (e.g. patients previously treated with any of the shorter regimen's drugs and in cases of documented or suspected resistance to one or more of them, with the exception of isoniazid) $[7,8]$.

The prevalence of resistance to the core drugs comprising the regimen in Europe and Latin America exceeded $60 \%$ for the first-line drugs (ethambutol and pyrazinamide), $50 \%$ for prothionamide and $40 \%$ for the two core drugs (fluoroquinolones and kanamycin) [1-8].

Existing evidence suggests that high-dose isoniazid is effective in the presence of the inhA gene (conferring low-level resistance to isoniazid but resistance to ethionamide/prothionamide) and, probably, of the kat $G$ (conferring intermediate-level resistance to isoniazid) [8]. Only two studies have provided data on genotypic resistance so far $[9,10]$.

The eligibility to the shorter regimen has not been studied widely in Latin America (with the exception of a single experience in Sao Paulo, Brazil [1]) and in Mexico (one of the largest countries in the region with relatively low drug-resistance prevalence).

As part of a collaborative TB project of the European Respiratory Society and ALAT (Latino-American Society of Thoracic Diseases), the aim of the present study was to assess the suitability to the shorter regimen in Mexico prior to its programmatic implementation, by performing drug susceptibility testing (DST) and genotypic resistance (GenoType MTBDR/MTBDR plus; Hain Lifescience, Neheren, Germany) in the Central TB Reference Institute (INER) in Mexico City.

The INER laboratory is validated within the WHO external quality control programme, and DST for firstand second-line drugs is performed by WHO-recommended methods using BACTEC and MGIT 960 (Becton Dickinson, Franklin Lakes, NJ, USA). Cohen's $\kappa$ statistic was calculated to assess inter-rater agreement between DST and genotypic results. Eligibility was evaluated according to three criteria (table 1): 1) no resistance to fluoroquinolones or injectable drugs, and no katG or inhA mutations (there is general agreement on this criterion, including WHO and the European Centre for Disease Prevention and Control (ECDC) $[7,8] ; 2$ ) as per criterion 1 plus no resistance to ethambutol (ECDC criterion) $[3,4] ; 3$ ) as per criterion 2 but no katG mutation (expert opinion only) [9].

Of the 120 cases evaluated, we excluded seven cases with disease caused by Mycobacterium bovis strains (three MDR-TB, two rifampicin-resistant (RR)-TB, one RR-TB with additional resistance to fluoroquinolones and one MDR-TB with resistance to second-line injectable drugs); given their intrinsic resistance to pyrazinamide, they are not eligible for the shorter regimen. Out of 112 confirmed consecutive MDR-TB regimen http://ow.ly/MOxJ30hXkjd

Cite this article as: Munoz-Torrico M, Salazar MA, Millán MdeJM, et al. Eligibility for the shorter regimen for multidrug-resistant tuberculosis in Mexico. Eur Respir J 2018; 51: 1702267 [https://doi.org/ 10.1183/13993003.02267-2017]. 
TABLE 1 Comparison of the results on phenotypic and genotypic resistance to antituberculosis drugs, Mexico, 2010-2017

\begin{tabular}{|c|c|c|c|}
\hline & $\begin{array}{l}\text { Phenotypic results } \\
\qquad(n=112)^{\#}\end{array}$ & $\begin{array}{l}\text { Genotypic results } \\
\qquad(\mathrm{n}=57)^{\#}\end{array}$ & к $(95 \% \mathrm{CI})$ \\
\hline Fluoroquinolone resistant & $26 / 111(23.4 \%)$ & $12 / 57(21.1 \%)$ & $0.894(0.751-1.000)$ \\
\hline Ofloxacin resistant & $26 / 111(23.4 \%)$ & & \\
\hline Moxifloxacin resistant & $8 / 49(16.3 \%)$ & & \\
\hline Injectable resistant & $13 / 111(11.7 \%)$ & $1 / 57(1.8 \%)$ & $0.226(-0.145-0.597)$ \\
\hline Amikacin resistant & $9 / 111(8.1 \%)$ & & \\
\hline Kanamycin resistant & $10 / 92(10.9 \%)$ & & \\
\hline Capreomycin resistant & $6 / 50(12.0 \%)$ & & \\
\hline Ethambutol resistant & $38 / 112(33.9 \%)$ & $19 / 57(33.3 \%)$ & $0.763(0.585-0.942)$ \\
\hline Isoniazid resistant & $97 / 112(86.6 \%)$ & $31 / 57(54.4 \%)$ & $0.597(0.402-0.793)$ \\
\hline katG and inhA mutations & & $1 / 57(1.8 \%)$ & \\
\hline katG mutation only & & $18 / 57(31.6 \%)$ & \\
\hline inhA mutation only & & $14 / 57(24.6 \%)$ & \\
\hline Pyrazinamide resistant & $46 / 110(41.8 \%)$ & & \\
\hline \multicolumn{4}{|c|}{ Eligible for the shorter regimen } \\
\hline Criterion 1 & $80(71.4 \%)$ & & \\
\hline Criterion 2 & $56(50.0 \%)$ & & \\
\hline Criterion 3 & $50(44.6 \%)$ & & \\
\hline
\end{tabular}

Criterion 1: eligible if no resistance to fluoroquinolones and injectables, and no katG or inhA mutations; criterion 2: eligible as per criterion 1 plus no resistance to ethambutol; criterion 3: eligible as per criterion 2 but no katG mutation (see text for details). " : the denominators vary as not necessarily all 112 strains underwent testing for all the drugs due to lack of reagents.

Mycobacterium tuberculosis MDR-TB cases analysed between 2010 and 2017 (originating from 17 out of 32 Mexican states), 57 underwent genotypic analysis (table 1). Phenotypic resistance to fluoroquinolones was $23.4 \%$ (26 out of 111 ) and genotypic $21.1 \%$ (12 out of 57 ; $\kappa$ 0.894, 95\% CI $0.751-1.000$ ); conversely, there was less resistance to the injectables (11.7\% and $1.8 \%$, respectively). Agreement between phenotypic and genotypic tests was good for ethambutol (33.9 versus 33.3\%, respectively; $\kappa$ 0.763, 95\% CI 0.585-0.942). A single case had combined katG and inhA mutations, 18 had katG only and $14 \operatorname{inh} A$ only (table 1).

According to criterion 1, $80(71.4 \%)$ MDR-TB patients would be eligible for the shorter regimen, 56 (50.0\%) as per criterion 2 and $50(44.6 \%)$ per criterion 3.

This is the first study comparing phenotypic and genotypic results in Latin America; it will help guide the adoption of the shorter regimen in Mexico. The study has limitations, as the sample size is relatively small and only half of the strains were subject to genotypic tests due to the limited availability of these tests in Mexico (few reference laboratories perform them). It is not easy to explain the low $\kappa$ value identified for injectables and further evidence is therefore needed. Although the study was performed in a single centre, $85(75.8 \%)$ patients or samples were referred from different states of Mexico with different prevalences of drug resistance.

Our results suggest that, although Mexico has a low prevalence of drug resistance, the shorter regimen cannot be introduced at the programmatic level without adequate testing with rapid methods and DST. Considering 1) the likelihood of drug esistance to ethambutol (most drug-resistant cases in Mexico are failures of a primary regimen including this drug), 2) the excellent DST proficiency results for ethambutol ( $>90 \%$ agreement between the INER laboratory and the supranational laboratory in Mexico City in 2016), and 3) the agreement between phenotypic and genotypic results on this drug, criterion 2 might potentially be the starting point to discuss the national adoption of the shorter regimen in the country.

Marcela Munoz-Torrico ${ }^{1}$, Miguel Angel Salazar ${ }^{1}$, Miriam de Jesús Mohedano Millán ${ }^{1}$, Jose A. Martínez Orozco ${ }^{2}$, Luis A. Narvaez Diaz ${ }^{2}$, Mariela Segura del Pilar $^{2}$, Dina Visca ${ }^{3}$ Lia D'Ambrosio ${ }^{4,5}$ Rosella Centis ${ }^{4}$ and Giovanni Battista Migliori $\oplus^{4}$

${ }^{1}$ Tuberculosis Clinic, National Institute of Respiratory Diseases of Mexico (INER), Mexico City, Mexico. ${ }^{2}$ Clinical Microbiology Laboratory, INER, Mexico City, Mexico. ${ }^{3}$ Pneumology Unit, Maugeri Care and Research Institute, IRCCS, Tradate, Italy. ${ }^{4}$ World Health Organization Collaborating Centre for Tuberculosis and Lung Diseases, Maugeri Care and Research Institute, IRCCS, Tradate, Italy. ${ }^{5}$ Public Health Consulting Group, Lugano, Switzerland. 
Correspondence: Giovanni Battista Migliori, WHO Collaborating Centre for TB and Lung Diseases, Fondazione S. Maugeri, Care and Research Institute, IRCCS, via Roncaccio 16, 21049 Tradate, Italy. E-mail: giovannibattista. migliori@icsmaugeri.it

Received: Nov 022017 | Accepted after revision: Dec 272017

Conflict of interest: None declared.

\section{References}

1 Sotgiu G, Tiberi S, D'Ambrosio L, et al. Faster for less, the new "shorter" regimen for multidrug-resistant tuberculosis. Eur Respir J 2016; 48: 1503-1507.

2 Dalcolmo M, Gayoso R, Sotgiu G, et al. Effectiveness and safety of clofazimine in multidrug-resistant tuberculosis: nationwide report from Brazil. Eur Respir J 2017; 49: 1602445.

3 van der Werf MJ, Hollo V, Ködmön C, et al. Eligibility for shorter treatment of multidrug-resistant tuberculosis in the European Union. Eur Respir J 2017; 49: 1601992.

4 van der Werf MJ, Ködmön C, Catchpole M. Shorter regimens for multidrug-resistant tuberculosis should also be applicable in Europe. Eur Respir J 2017; 49: 1700463.

5 Chee CBE, KhinMar KW, Sng LH, et al. The shorter multidrug-resistant tuberculosis treatment regimen in Singapore: are patients from South-East Asia eligible? Eur Respir J 2017; 50: 1700753:

6 Javaid A, Ahmad N, Hayat Khan A, et al. Applicability of the World Health Organization recommended new shorter regimen in a multidrug-resistant tuberculosis high burden country. Eur Respir J 2017; 49: 1601967.

7 Falzon D, Schunemann HJ, Harausz E, et al. World Health Organization treatment guidelines for drug-resistant tuberculosis, 2016 update. Eur Respir J 2017; 49: 1602308.

8 Sotgiu G, Tiberi S, Centis R, et al. Applicability of the shorter 'Bangladesh regimen' in high multidrug-resistant tuberculosis settings. Int J Infect Dis 2017; 56: 190-193.

9 Chesov D, Ciobanu N, Lange C, et al. Lack of evidence of isoniazid efficacy for the treatment of MDR/XDR-TB in the presence of the katG 315T mutation. Eur Respir J 2017; 50: 1701752.

10 Barry PM, Lowenthal P, True L, et al. Benefit of the shorter MDR TB treatment regimen in California and modified eligibility criteria. Am J Respir Crit Care Med 2017; 196: 1488-1489. 